\title{
The Western Front
}

\author{
ANTHONY G. O'FARRELL
}

In homage to T.T. West.

\begin{abstract}
This is an expanded version of a talk given (under the title Ars Longa - Im Westen nichts Neues) at the symposium to honour T.T. West, held in Trinity College on 19-20 December 2005. It describes some problems posed by Trevor West, arising from his work, or related to it.
\end{abstract}

\section{INTRODUCTION}

Finding a friend is like winning the lottery, only better. Moolah doesn't make you happy, as friends do. The resemblance lies in the rarity of the thing.

Trevor is such a colourful and luminous character that all others seem dull and gray beside him. We shine not. We are visible, at best, thanks to his illumination. I'm not sure what caused him to favour me with his attention, in the first place. Perhaps it was just that he needed a place to sleep, one evening in L.A., and picked the nearest Irishman as his host. Whatever the reason, he certainly got my attention, with his uniquely engaging style of conversation, and more than earned his supper.

Thereafter, he took me under his wing, maintained a steady and amusing correspondence, kept me informed about jobs in Ireland, coached me about all manner of practical realities, and in due course saw me safely established in my present chair at 28 and elected to the Academy at 33. Basically, his method of orchestrating these things was to slip me in quickly under their guard, before they had a chance to realise what a big mistake they were making. He always kept matters straight between the two of us. His letter (from Seanad Éireann) of 1-7-1975 reads:

Supported by SFI grant 05/RFP/MAT0003. 
Well done O'Farrell,

Remember tho' you now really are a Professor at Maynooth You are still a uniformly algebraic $\mathrm{b}-\mathrm{x}$.

West

His letters over the years came with a stream of questions, and I suppose my side of the bargain may have been that I was supposed to give these the attention they deserved. In practice, I answered the easy ones straight away, and put the others to one side for later study.

Looking through the file (like the Minister for Justice, I have a file on all of you) in preparation for this meeting, I now find all these problems looking up at me, patiently, if rather reproachfully, awaiting their day in the light. The distressing thing about this is that the file also has copies of some answers to the easy problems, and on scanning these I find them barely intelligible, laced with references to books I've forgotten reading, and marked by the unconscious hubris of youth.

Plainly, I may never get to give these questions their due. My present preoccupation with involutions and reversibility in groups of maps looks set to keep me occupied for some time. So it seems a good idea to ventilate some of these problems from the "Western Front".

As professional Mathematicians, it is our honourable and decent occupation, and our pleasure, to identify and address problems that are interesting and hard. When I was young I gathered questions in the hope that one day I would get round to them myself. Later, beginning to understand the scale of the task, I hoped to live to see others solve them. Now, I take the view espoused by Hobson. In his little book [4] about squaring the circle, he talks about the remarkable patience and persistence of the mathematical community considered as a single organism, which ground away for well over two thousand years until it finally resolved this ancient enigma. In feeding the following problems to the maw of Hobson's organism, I am confident that they will be resolved in due course, but don't expect that it will take quite so long, unless a new dark age intervenes and closes off the present golden age of Mathematics. 


\section{Spectral Radius Algebras}

This involves a problem that I find particularly interesting.

A (complex) Banach algebra is a complete normed algebra [2] over the field $\mathbb{C}$ of complex numbers. The main examples are algebras of functions (with pointwise multiplication), algebras of linear operators (with composition as the multiplication), convolution algebras of functions and measures on groups, and variants of these, such as truncated convolution algebras on semigroups. The spectral radius of an element $x$ of a Banach algebra $A$ is the nonnegative number $r_{A}(x)$ given by

$$
r_{A}(x)=\lim _{n \uparrow \infty}\left\|x^{n}\right\|^{1 / n} .
$$

When $A$ has an identity, this equals

$$
\sup \{|\lambda|: \lambda \in \sigma(x)\},
$$

where $\sigma_{A}(x) \subset \mathbb{C}$ is the spectrum of $x$ :

$$
\sigma_{A}(x)=\{\lambda \in \mathbb{C}: \lambda \cdot 1-x \text { is not invertible in } A\} .
$$

2.1. Quotient Algebras. The story begins with a study by Smyth and West [11] on the spectral radius in quotient algebras. Let $A$ be a Banach algebra, and $I$ be a closed two-sided ideal in $A$. For an element $x \in A$, let $r(x)$ denote the spectral radius $r_{A}(x)$ of $x$ (with respect to $A)$, and $r(x+I)$ denote the spectral radius $r_{A / I}(x+I)$ of the coset $x+I$ (with respect to $A / I$ ). In general,

$$
r(x+I) \leq \inf _{y \in I} r(x+y) .
$$

They called $A$ an $S R$-algebra if equality holds for each closed ideal $I$ and each $x \in A$. They asked:

Problem 2.1. Characterise the SR algebras.

They gave interesting examples of SR-algebras and of non-SRalgebras. See [5] for further detail and references. In the latter paper, they gave an elegant new proof of the fact (first shown by Pedersen in 1976) that every $\mathrm{C}^{*}$-algebra is SR. This proof is based on the formula they discovered:

$$
r(x)=\inf _{a=a^{*} \in A}\left\|e^{a} x e^{-a}\right\|,
$$

which is valid for each element $x$ belonging to a unital $\mathrm{C}^{*}$-algebra $A$.

Smyth and West also showed that each Riesz algebra is an SRalgebra. The Riesz algebras $A$ may be described as those Banach 
algebras for which the nonzero part of the spectrum of every element forms a countable discrete set (although Smyth's original definition is purely algebraic). Thus they include many natural algebras of operators.

2.2. The Commutative Case. Now consider the question for commutative Banach algebras. Denoting the character space, or maximal ideal space of $A$ by $\Sigma(A)$, the Gelfand transform maps $A$ homomorphically onto an algebra $\hat{A}$ of continuous functions on $\Sigma(A)$, a subalgebra of the uniform algebra $C(\Sigma(A))$, i.e. we have an exact sequence

$$
0 \rightarrow \operatorname{Rad}(A) \rightarrow A \rightarrow \hat{A} \rightarrow 0 .
$$

The Gelfand map $x \mapsto \hat{x}$ is given by

$$
\hat{x}(\phi)=\phi(x), \quad \forall \phi \in \Sigma(A) .
$$

It is continuous, but not in general isometric, and its kernel is the Jacobson radical, $\operatorname{Rad}(A)$.

The spectral radius $r(x)$, in this context, is just the sup norm of $\hat{x}$ on $\Sigma(A)$.

Smyth and West noted that if $\hat{A}$ is a dense subset of $C(\Sigma(A))$, then $A$ is an SR-algebra.

What about the converse?

In particular, what about the case of uniform algebras? (For a uniform algebra, the Gelfand transform is an isometry, i.e. $A$ may be identified with $\hat{A}$, as a Banach algebra.)

Problem 2.2. Which uniform algebras are SR algebras? If $A$ is a uniform $S R$ algebra, is $A=C(X)$, for some $X$ compact Hausdorff?

This question connects with some very old concerns. Let's put it into a wider context.

2.3. Closed Restrictions. Suppose we are given a uniform algebra $A \subset C(X)$, where $X$ is a compact Hausdorff space. We assume that $A$ separates points on $X$, that $1 \in A$, and that $A$ is uniformly closed. We do not assume that $X$ is $\Sigma(A)$. We may take it, without loss in generality, that $X \subset \Sigma(A)$, and that $X$ contains the Shilov boundary $\operatorname{Sh}(A)$ [2]. In practice, one is usually interested in the cases $X=\Sigma(A)$ and $X=\operatorname{Sh}(A)$.

Associated to $A$, we may identify the following families of subsets of $X$ : 
$\mathcal{W}$ : The family of weak-star closed subsets of $X$.

$\mathcal{C}$ : The family of $A$-convex subsets of $X ; E \in \mathcal{C}$ if and only if

$$
E=\left\{a \in X:|f(a)| \leq \sup _{E}|f|, \forall f \in A\right\} .
$$

$\mathcal{H}$ : The family of (relatively) hull-kernel closed sets; $E \in \mathcal{H}$ if and only if

$$
E=\{a \in X: f(a)=0 \text { whenever } f \in \operatorname{ker}(E)\},
$$

where

$$
\operatorname{ker}(E)=\{f \in A: f \mid E=0\},
$$

where $f \mid E$ denotes the restriction of $f$ to $E$.

$\mathcal{R}$ : The family of $A$-convex sets $E$ such that the algebra $A \mid E$ of all restrictions $f \mid E$, for $f \in A$, is uniformly-closed.

$\mathcal{R}_{1}$ : The family of $A$-convex sets $E$ such that the quotient $A / \operatorname{ker} E$ is isometric to the restriction algebra, i.e. given $f \in A$ and $\epsilon>0$, there exists $g \in A$ such that $g|E=f| E$ and $\sup _{X}|g| \leq(1+\epsilon) \sup _{E}|f|$.

I: The family of interpolation sets; $E \in \mathcal{I}$ if $E$ is weak-star closed $E$ and $A \mid E=C(E)$.

$\mathcal{I}_{1}$ : The family of isometric interpolation sets; $E \in \mathcal{I}_{1}$ means that $C(E)$ is isometric to $A / \operatorname{ker} E$.

$\mathcal{P}$ : The family of p-sets in $X$, i.e. intersections of peak sets. That $P$ is a peak set means that there is a function $f \in A$ with $f=1$ on $P$ and $|f|<1$ off $P$.

$\mathcal{P} \cap \mathcal{I}$ : The family of p-interpolation sets.

The relationship between these families is expressed in the following diagram, in which each arrow is an inclusion map [9]:

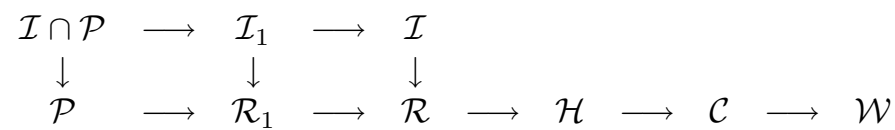

The non-obvious aspects of this pattern of inclusions are results of Glicksberg and Bernard. Each inclusion may be proper.

Various classes of uniform algebras are defined by requiring equality in one or more of the inclusions.

For instance, the class of regular uniform algebras is characterised by $\mathcal{H}=\mathcal{W}$ when $X=\Sigma(A)$. The Murphy-West class of SR uniform algebras are precisely those for which $\mathcal{R}_{1}=\mathcal{H}$ when $X=\Sigma(A)$. 
Thus the Murphy-West problem is just one of the equality problems posed by the diagram, for the case $X=\Sigma(A)$. The equality problems are quite different, but no less interesting, for the case $X=\operatorname{Sh}(A)$.

To appreciate the distinction, consider the example of the disc algebra $A(\mathbb{D})$, which has as Shilov boundary the circle $\mathbb{S}^{1}$, and as character space the closed unit disc. In case $X=\mathbb{S}^{1}$, the diagram has just two distinct families of sets. $\mathcal{P} \cap \mathcal{I}=\mathcal{H}$, and $\mathcal{C}=\mathcal{W}$. The main content of this remark is the celebrated Rudin-Carleson Theorem [2]. In case $X=\operatorname{clos}(\mathbb{D})$, the family of hull-kernel closed sets is larger than $\mathcal{R}_{1}$. For instance, $\left\{-\frac{1}{2}, \frac{1}{2}\right\}$ does not belong to $\mathcal{R}_{1}$. (The paper [9] considers the Shilov boundary case and includes an extension of Rudin-Carleson to a wider context.)

We state the general problem:

Problem 2.3. For each pair of families in the diagram, and for both cases $X=\Sigma(A)$ and $X=\operatorname{Sh}(A)$, characterise equality.

Some equalities in the diagram define the class of $C(X)$ algebras, i.e. of commutative $C^{*}$-algebras. Glicksberg showed in 1963 [3] that this is the case for the equality $\mathcal{R}=\mathcal{W}$. Thus if we assume $A$ regular, then $A$ is an SR-algebra if and only if $A=C(\Sigma(A))$. But what about algebras $A$ that are not assumed regular a priori? One has the following:

Theorem 2.1. Suppose that $A$ is a uniform algebra on its maximal ideal space $X=\Sigma(A)$, and is an $S R$-algebra. Then

(1) Every quotient of $A$ by a closed ideal is a uniform algebra;

(2) A admits no nonzero bounded point derivations; and

(3) All the Gleason parts of $A$ are trivial (i.e. consist of singletons).

A bounded point derivation is a continuous linear functional $\partial$ : $A \rightarrow \mathbb{C}$ such that

$$
\partial(f g)=f(a) \partial(g)+\partial(f) g(a)
$$

holds for some point $a \in X$ and all $f, g \in A$. A bounded point derivation annihilates the ideal $M^{2}$, where $M$ is the maximal ideal $\operatorname{ker}(\{a\})$ corresponding to the associated point $a$. It also annihilates constants, so if it not zero, then the ideals $M$ and $\operatorname{clos} M^{2}$ differ. 
The Gleason parts [2] of $A$ are subsets of $\Sigma(A)$. They are the equivalence classes under the Gleason equivalence relation

$$
a \equiv b \quad \Longleftrightarrow \sup _{f \in \operatorname{Ball}(A)}|f(a)-f(b)|<2 .
$$

Proof. (1) follows from the isometric isomorphism

$$
A \mid E \simeq A / I,
$$

valid whenever $E=\operatorname{hull}(I)$ for a closed ideal $I$. This also shows that there can only be one closed ideal to each hull ("spectral synthesis"), and hence there can be no bounded point derivations, because the ideals $M$ and $\operatorname{clos} M^{2}$ described above both have hull $\{a\}$.

The hypothesis may be rephrased as saying that whenever $E$ is a hull and $f \in A$, the restriction $f \mid E$ has an almost-norm-preserving extension to $X$ in $A$. In particular, each function defined on a finite subset of $X$ has an almost-norm-preserving extension to $X$ in $A$. Applying this to two-point sets, we deduce (3).

This shows that if a uniform SR-algebra is not a $C(X)$, then it is a rather odd object. However, properties (2) and (3) are not, by themselves, enough to characterise $C(X)$, as was shown by famous examples of McKissick and of Cole. Indeed one is reminded by this of the oldest open problem about uniform algebras:

Problem 2.4. If $A$ is a uniform algebra and $\Sigma(A)=[0,1]$, is $A=$ $C([0,1])$ ?

So, following Polya's maxim [10] that whenever there is a problem you can't solve, then there is always an easier problem you can't solve, it is tempting to pose this:

Problem 2.5. Suppose that $\Sigma(A)=S h(A)=[0,1]$, and $A$ is an $S R$ uniform algebra. Must $A=C([0,1])$ ?

Indeed, as far as I know, this is open even when $A$ is generated by just two elements. Putting it another way:

Problem 2.6. Does there exist a polynomially-convex arc $\Gamma \in \mathbb{C}^{2}$ such that the uniform closure $P(\Gamma)$ on $\Gamma$ of the algebra of analytic polynomials is an SR-algebra, has $\Gamma$ as its Shilov boundary, and is $\operatorname{not} C(\Gamma)$ ? 


\section{Uniformly-Distributed SEQUEnCES}

If $\lambda$ is a complex number of modulus one, then

$$
\frac{1}{n} \sum_{k=1}^{n} \lambda^{k} \rightarrow \begin{cases}0 & , \quad \lambda \neq 1 \\ 1 & , \quad \lambda=1 .\end{cases}
$$

Problem 3.1. For what sequences $\left\{j_{k}\right\}$ of positive integers does

$$
\frac{1}{n} \sum_{k=1}^{n} \lambda^{j_{k}} \rightarrow \begin{cases}0 & , \quad \lambda \neq 1 \\ 1 & , \quad \lambda=1 ?\end{cases}
$$

This came from West in July, 1993, motivated by "some problem in ergodic theory".

\section{Supports of Measures}

Let $X$ be a compact Hausdorff space. A Radon measure on $X$ is, by definition, an element of the dual space of $C(X, \mathbb{R})$. Such a measure may be identified with a suitable set function $\mu: 2^{\mathbb{R}} \rightarrow \mathbb{R}$, and the support of $\mu$ is defined as the complement of the largest open set $U$ for which $\mu(E)=0$ whenever $E \subset U$. We say that $X$ satisfies the countable chain condition (ccc) if each pairwise-disjoint family of open sets is countable.

Murphy and West noted [7], Prop. 1] that if there exists a Radon measure on $X$ with support equal to $X$, then $X$ satisfies the ccc.

The question is whether or not the converse holds.

Problem 4.1. Suppose $X$ is a compact Hausdorff space satisfying the countable chain condition. Does there exist a Radon measure with support equal to $X$ ?

The answer may depend on your set theory.

\section{Basic and Removable Spectrum}

For a commutative Banach algebra $A$ with unit, recall that $\sigma_{A}(x)$ denotes the spectrum of an element $x \in A$. If $A$ is isometrically and algebraically imbedded in a larger algebra $B$, then

$$
\sigma_{B}(x) \subset \sigma_{A}(x), \quad \text { and } \quad \operatorname{bdy}\left(\sigma_{A}(x)\right) \subset \operatorname{bdy}\left(\sigma_{B}(x)\right) .
$$

Let $A(x)$ denote the closure of the subalgebra with unit $\mathbb{C}[x]$ generated by $x$ in $A$. The spectrum of $x$ with respect to $A(x)$ is called the basic spectrum of $x$, and denoted $\sigma^{\prime}(x)$. This spectrum 
is polynomially-convex, so it comprises $\sigma_{A}(x)$ together with all its holes.

The problem of removing the interior of the spectrum [6] has to do with finding (if possible) an isometric extension $B$ of $A$ such that $\sigma_{B}(x)=\operatorname{bdy}\left(\sigma^{\prime}(x)\right)$. For instance, if $A=A(\mathbb{D})$, the disc algebra, then the problem is solved by taking $B=C\left(\mathbb{S}^{1}\right)$.

The problem is related to so-called approximate divisors of zero (ADZ's). An element $z \in A$ is an $\mathrm{ADZ}$ in $A$ if there exist $y_{n} \in A$, for $n=1,2, \ldots$, such that

$$
\left\|y_{n}\right\|=1 \quad \text { and } \quad y_{n} z \rightarrow 0 .
$$

In general, it is true that

$$
\lambda \in \operatorname{bdy}\left(\sigma_{A}(x)\right) \Rightarrow \lambda-a \text { is an } \mathrm{ADZ} \Rightarrow \lambda \in \sigma_{A}(x) .
$$

Thus it is obvious that $\lambda$ cannot be removed from the spectrum in any extension if $\lambda-a$ is an ADZ. Shilov and Arens showed that the converse holds. Thus the essential problem becomes:

Problem 5.1. Characterise those CBAs, A, (topologically) generated by a single $x \in A$, such that $\lambda-x$ is an $A D Z$ in $A$ only when $\lambda \in \operatorname{bdy}\left(\sigma_{A}(x)\right)$

It is known that all uniform algebras and certain algebras generated by an operator have this property, and there are examples of algebras that do not [6].

There is a related question about the cortex of $A$, the set of characters on $A$ that extend to characters on each extension $B$ of $A$ : If $x$ is an ADZ, does the Gelfand transform $\hat{x}$ vanish somewhere on the cortex of $A$ ?

\section{Power-Bounded Operators and Semigroups}

Given a bounded linear operator $T$ on a Banach space $X$, it generates a semigroup $\langle T\rangle$, an algebra $\mathbb{C}(T)$, and a semialgebra $A(T)=\mathbb{Z}^{+}[T]$. We say that $T$ is power-bounded if $\langle T\rangle$ is bounded.

West's work on weakly-compact groups and locally-compact semialgebras of operators relates these compactness properties to one another and to properties of the peripheral spectrum of a powerbounded operator.

This prompts me to mention another open problem in one complex variable that arose in connection with the study of power-bounded operators. The following Tauberian theorem is proved in [1]: 
Theorem 6.1. Let $f(z)=\sum_{n=0}^{\infty} a_{n} z^{n}$ have radius of convergence 1 , let $E \subset \mathbb{S}^{1}$ be the set of essential singularites of $f$ on $S^{1}$, suppose $E$ has length (1-dimensional Hausdorff measure) zero and suppose that there exists $M<+\infty$ bounding all the partial sums of the series on $E$ :

Then

$$
\left|\sum_{n=0}^{N} a_{n} \zeta^{n}\right| \leq M, \quad \forall N \in \mathbb{N}, \forall \zeta \in E
$$

$$
\sum_{n=0}^{\infty} a_{n} z^{n} \text { converges to } f(z)
$$

for each $z \in \mathbb{S}^{1} \sim E$.

As an immediate consequence, the hypotheses imply that $a_{n} \rightarrow 0$.

Problem 6.1. Suppose that $f$ is as in the theorem, except that we drop the assumption that $E$ have length zero. Must $a_{n} \rightarrow 0$ ?

It follows from theorems of Fatou and Carleson that the answer is yes in the case $E=S^{1}$.

\section{CODA}

Present and future Irish Mathematicians owe a great debt to Trevor for his long and successful efforts to raise the national game. Particularly noteworthy are his part [13] in the formation of the Irish Mathematical Society, and his work on the Mathematical Proceedings of the Royal Irish Academy. One must also acknowledge his encouragment of younger colleagues, both in Mathematics and in other sports, his wider contribution in national politics, and his outstanding account [12] of the life and times of Horace Plunkett, who (like Trevor) did much to help bridge the religious divide in Ireland.

\section{REFERENCES}

[1] G.R. Allan, A.G. O'Farrell and T.J. Ransford. A Tauberian theorem arising in operator theory. Bull. London Math. Soc. 19 (1987), 537-45.

[2] T.W. Gamelin. Uniform Algebras. Chelsea. New York 1984.

[3] I. Glicksberg. Function algebras with closed intersections. Proc. Amer. Math. Soc. 14 (1963), 158-61.

[4] E. Hobson. Squaring the Circle \& Other Monographs. Chelsea. New York 1988.

[5] G.J. Murphy and T.T. West. Spectral radius formulae. Proc. Edinburgh Math. Soc. 22 (1979), 271-275. 
[6] G.J. Murphy and T.T. West. Removing the interior of the spectrum-Shilov's example. Comm. Math. Univ. Carolinae 21 (1980), 421-431.

[7] T. Murphy and T.T. West. Inner products in Banach spaces and supports of Radon measures. Proc. Roy. Ir. Acad. 69A (1970), 55-61.

[8] A.G. O'Farrell. Restriction Algebras. Proc. Roy. Ir. Acad. 82A (1982), 105108.

[9] A.G. O'Farrell. Restrictions of Uniform Algebras. In: Complex Analysis and Applications '81. Sofia. 1984, pp. 161-166.

[10] G. Polya. How to Solve It. Princeton University Press, 1971.

[11] M.R.F. Smyth and T.T. West. The spectral radius formula in quotient algebras. Math. Z. 145 (1975), 157-161.

[12] T.T. West. Horace Plunkett: Cooperation and Politics, an Irish Biography. Catholic University of America Press. Washington 1986.

[13] T.T. West. The origins of the Irish Mathematical Society. Bull. Ir. Math. Soc. 51 (2003), 73-75.

Anthony G. O'Farrell,

Department of Mathematics

National University of Ireland,

Maynooth, Co. Kildare, Ireland

anthonyg.ofarrell@gmail.com

Received on 25 January 2006. 